\title{
A review of current methods for assessing hemostasis in vivo and introduction to a potential alternative approach
}

\author{
Mallory R. Scolaa, ${ }^{a}$, Leslie M. Baggesen ${ }^{a}$, Tim C. Nichols ${ }^{b, c}$, Nigel S. Key ${ }^{c, d}$, and Caterina M. \\ Gallippi ${ }^{\mathrm{a}}$ \\ a Joint Department of Biomedical Engineering, The University of North Carolina at Chapel Hill, \\ Chapel Hill, NC, USA \\ ${ }^{b}$ Department of Pathology and Laboratory Medicine, The University of North Carolina at Chapel \\ Hill, Chapel Hill, NC, USA \\ ${ }^{c}$ Department of Medicine, The University of North Carolina at Chapel Hill, Chapel Hill, NC, USA \\ d Division of Hematology, The University of North Carolina at Chapel Hill, Chapel Hill, NC, USA
}

\begin{abstract}
A validated method for assessing hemostasis in vivo is critical for testing the hemostatic efficacy of therapeutic agents in preclinical animal models and in patients with inherited bleeding disorders, such as von Willebrand disease (VWD) and hemophilia A, or with acquired bleeding disorders such as those resulting from medications or disease processes. In this review, we discuss current methods for assessing hemostasis in vivo and the associated challenges. We also present ARFI-Monitored Hemostatic Challenge; a new, potentially alternate method for in vivo hemostasis monitoring that is in development by our group.
\end{abstract}

\section{Keywords}

Hemostasis; Hemostatic challenge; Canine model; Acoustic Radiation Force Impulse (ARFI); ultrasound imaging; Bleeding disorders; Soft-tissue bleeding

\section{Introduction}

Bleeding is a frequent and deadly complication of hematologic and cardiovascular disorders - inherited or acquired. Inherited bleeding disorders, such as von Willebrand disease (VWD) and hemophilia A, result from genetic deficiencies in plasma coagulation proteins and must be managed by life-long intravenous infusion of therapeutic clotting factor replacement products [1]. For plasma-derived products, despite the introduction of viral inactivation techniques that have eliminated the risk of HIV transmission, there are ongoing concerns about other transmissible agents (e.g., prions) that may not be eliminated [2-7]. Fortunately, the risk of infection from recombinant products seems negligible. Acquired bleeding disorders most frequently arise in subjects with atherothrombotic disease from the combined use of currently approved anticoagulant (Coumadin, dabigatran etexilate, rivaroxaban), and

\footnotetext{
(c) 2012 Published by Elsevier Ltd.

*Corresponding author. cmgallip@bme.unc.edu (M.R. Scola)..

Conflict of interest statement

Research in the area of the ARFI Monitored Hemostatic Challenge was supported by grant HL63098 and unrestricted grants from

Marine Polymer Technologies, Inc. and Novo Nordisk.
} 
antiplatelet (aspirin, clopidogrel, prasugrel) agents. This complication is likely to become even more prevalent with the aging population, and it accounts for a majority of drug-related emergency hospitalizations [8]. While vitamin $\mathrm{K}$ or plasma products can reverse Coumadin's effect and are routinely used to treat or prevent bleeding associated with Coumadin use, the rapidly growing number of antithrombotic agents with no proven reversal therapies constitute a common clinical challenge, and major hemorrhage is arguably now the ratelimiting step in the development of even more effective antithrombotic strategies.

Fortunately, innovative approaches such as gene therapy and protein engineering [9-11] are in development to control bleeding from VWD and hemophilia. The novel proteins such as recombinant factor VIIa (rFVIIa) are also often used to reverse bleeding due to antithrombotic therapy. This choice is in part because current expert statements on the management of bleeding induced by established and emerging anticoagulants usually include a suggestion to use rFVIIa [12,13], despite the paucity of clinical evidence or preclinical data to support this recommendation $[14,15]$. However, translating these approaches to clinical trials requires a reliable diagnostic test that directly detects early bleeding and confirms hemostasis in vivo.

\section{Plasma and Whole Blood -Based Coagulation Factor Activity Assays}

Importantly, plasma-based coagulation factor activity assays are helpful surrogates for hemostasis but do not directly measure hemostasis in vivo. These include the standard aPTT [16], two stage and chromogenic assays. Whole blood-based assays include the whole blood clotting time (WBCT) which is a modification of the Lee-White clotting time using two siliconized glass tubes (Vacutainer ${ }^{\mathrm{TM}} \# 6431$, Becton-Dickinson, Rutherford, NJ) in a $28^{\circ} \mathrm{C}$ water bath $[17,18]$. One $\mathrm{ml}$ of whole blood is drawn and $0.5 \mathrm{ml}$ blood is distributed into each dry tube. A timer is started. After one minute, one tube is tilted every $30 \mathrm{sec}$, the other left undisturbed. When a clot forms in the tilted tube, the second tube is then tilted every $30 \mathrm{sec}$ until a clot forms. The time for formation of a fully gelled clot in the second tube is recorded as the WBCT.

\section{Current In Vivo Hemostasis Assays in Large Animal Models}

Pre-clinically, conventional in vivo tests to assess the hemostatic efficacy of therapeutic agents include the primary saline bleeding time $\left(1^{\circ} \mathrm{SBT}\right)$ in VWD dogs and the primary cuticle bleeding time $[19,20]$, the gingival biopsy bleeding time [21], and the secondary cuticle bleeding time ( $\left.2^{\circ} \mathrm{CBT}\right)$ [22-24] in hemophilia A dogs. Several variations of the primary bleeding time have been used to detect disorders of primary hemostasis such as von Willebrand disease (VWD) [25,26] and monitor the response to therapy. We have used the $1^{\circ} \mathrm{SBT}$ described by Mertz [27] in canine and porcine models of type 3 VWD [9,28]. This test is done with the tip of the ear immersed in $37^{\circ} \mathrm{C} 0.9 \%$ saline for 2 minutes, then the tip is transfixed with a scalpel blade over $\sim 0.5 \mathrm{~cm}$, the cut ear tip is resub-merged in the warm saline, and the time to cessation of bleeding is recorded. A normal value is defined as $<5$ minutes and abnormal value is defined as $>15$ minutes. In the primary and secondary cuticle bleeding time tests, excess hair is shaved away from the toe and the paw is immersed in 37 ${ }^{\circ} \mathrm{C} 0.9 \%$ saline for 2 minutes. Using a guillotine-type of nail cutter, the primary cut is made at the juncture of the nail with the nail bed or "quick." The bleeding nail is immersed in 37 ${ }^{\circ} \mathrm{C} 0.9 \%$ saline and the endpoint is the time that bleeding convincingly stops for a full minute and is called the Primary Cuticle Bleeding Time [19,20]. For the Secondary Cuticle Bleeding Time, the injured surface is subjected to a second guillotine cut 2 hours after the first, the bleeding nail is again immersed in $37^{\circ} \mathrm{C} 0.9 \%$ saline, bleeding is visually monitored and the endpoint is the time that bleeding convincingly stops for a full minute [29-32]. For gingival or buccal mucosa bleeding time tests, various methods for wounding 
oral mucosa to induce bleeding have been described and, in general, the time to cessation of bleeding is reported as the gingival or buccal mucosa bleeding time [21,26,33].

Despite many years of use, significant problems with reproducibility persist for these current in vivo tests. Table 1 shows results of performing the $1^{\circ}$ SBT [27,34], in 11 dogs with VWD and the $2^{\circ} \mathrm{CBT}$ in 20 dogs with hemophilia A [9,28]. Recall a normal value is defined as $<5$ $\mathrm{min}$, and abnormal value is defined as $>15 \mathrm{~min}$. The $1^{\circ} \mathrm{SBT}$ was $>15 \mathrm{~min}$ in $7 / 11 \mathrm{dogs}$ on the first try, reproducible in only 4 of these 7 , and excessive bleeding and unclear endpoints were common due to waxing and waning rates of bleeding (row a). The $2^{\circ} \mathrm{CBT}$ was $>15$ min on the first attempt in fewer than half the animals tested, the test was reproducibly long in only half the dogs tested twice, and four dogs had excessive bleeding that necessitated stopping the test prematurely. Eight dogs also had unclear endpoints due to the sudden resurgence of bleeding immediately after stopping or appearing to stop (row b). Additional complications included inadequate anatomy for the prescribed cuticle incisions and dogs licking, scratching and ambulating during the observation period. The inherent variability in both $1^{\circ} \mathrm{SBT}$ and $2^{\circ} \mathrm{CBT}$ requires screening and exclusion of many dogs from entering studies, necessitating use of more animals. Moreover, as these tests involve monitoring bleeding from induced injury at the nail cuticle or ear tip, neither assesses a clinically relevant bleeding location or hemostatic challenge.

\section{Novel ARFI-Monitored Hemostatic Challenge}

We have recently developed the ARFI-Monitored Hemostatic Challenge, in which bleeding response to a peripheral vascular puncture is monitored by Acoustic Radiation Force Impulse (ARFI) imaging methods. ARFI ultrasound noninvasively differentiates tissue mechanical properties by tracking dynamic tissue displacement in response to impulsive acoustic radiation force excitation [35]. A detailed description of the ARFI-Monitored Hemostatic Challenge is provided in [36]. Notably, the ultrasound transducer is positioned above a punctured peripheral vessel (Fig. 1) and custom software is used to isolate blood pixels from the surrounding soft tissue pixels. Once blood pixels are identified, the crosssectional area of hemorrhage can be measured in each of several serially acquired ARFI images, from which bleeding rate (BR) and time to hemostasis (TTH) are assessed, as shown in Fig. 2.

Pre-clinically, the ARFI-Monitored Hemostatic Challenge is implemented by puncturing capillaries and a small ( $2 \mathrm{~mm}$ diameter) vein in hind limb muscle approximately $2 \mathrm{~cm}$ below the skin surface [36]. Table 2 shows the BR and TTH results of performing the ARFIMonitored Hemostatic Challenge in the following series of dogs: normal $(n=6)$, naïve hemophilia A monitored for 30 minutes of bleeding then infused with canine FVIII to $11.3 \pm 1.8 \%$ activity $(n=8)$ to prevent formation of large hematomas, hemophilia $A$ expressing canine factor FVIIa at $1.3-2 \mu \mathrm{g} / \mathrm{ml}(\mathrm{cFVIIa}, \mathrm{n}=3)$ [37], naïve VWD (n=6), and VWD infused with von Willebrand Factor (VWF) to a level of $7.1 \pm 1.7 \%$ of normal VWF:Ag ( $n=4)$. Relative to abnormal primary hemostasis, ARFI-derived BR was markedly increased in naïve VWD dogs compared to normal dogs by a factor of $\sim 2$. Note that prophylactic infusion of VWF from $<1 \%$ to a level of $7.1 \pm 1.7 \%$ of normal VWF: Ag was associated with a mean ARFI-derived bleeding rate of less than one-third that in naïve VWD and not statistically significantly different than normal. FVIII activity (Coamatic FVIII activity, Chromogenix), historically higher in VWD dogs than type 3 VWD humans [38], was $64 \pm 7.4$ pre and $57 \pm 22.4$ post infusion expressed as a $\%$ of normal canine plasma. These FVIII activity levels strongly suggest that this reduction in bleeding rate is due to correction of a VWF-mediated platelet defect of primary hemostasis and not FVIII deficiency. Mean ARFI-derived TTH in naïve and prophylactically-treated VWD dogs were not significantly different than normal. 
Relative to abnormal secondary hemostasis, ARFI-derived TTH was significantly longer in naive hemophilia A compared to normal dogs. Administration of FVIII to $11.3 \pm 1.8 \%$ (Coamatic FVIII activity, Chromogenix) in an "on-demand" fashion to treat the bleeding hemophilia A dogs shortened the time to hemostasis, but the difference was significant relative to normal dogs. Notably, transgene expression of canine FVIIa at $1.3-2 \mu \mathrm{g} / \mathrm{ml}$ (a therapeutic level) [37] shortened the time to hemostasis relative to naïve hemophilia A and was not significantly different than normal. ARFI-derived bleeding rates calculated over the first $30 \mathrm{~min}$ following puncture were not significantly different between normal, naïve hemophilia A, hemophilia A treated with FVIII "on-demand", or hemophilia A dogs expressing cFVIIa.

The data of Table 2 demonstrate that ARFI Monitored hemostatic Challenge yields outcomes that are consistent with expectations for the various bleeding phenotypes and associated treatments examined: 1) statistically significantly faster rates of bleeding in von Willebrand's disease, a disorder of primary hemostasis, 2), statistically significantly longer times to hemostasis in hemophilia A, a disorder of secondary hemostasis, and 3) full or partial correction of bleeding phenotype with treatment. Taken together, these data strongly support that ARFI-Monitored Hemostatic Challenge is relevant for assessing hemostasis and responses to treatment in vivo in inherited disorders of both primary and secondary hemostasis. In order to develop the ARFI-Monitored Hemostatic Challenge as a validated in vivo hemostasis assay, its accuracy and reproducibility must be established. While not yet completed at the time of publication, these properties are being assessed. We have not systematically looked at complications, but they appear limited to a small hematoma $(<2$ $\mathrm{cm}$ ). In addition, no dog has required additional blood products after completion of the experimental protocol. Although we have only applied our methods on relevant dog models pre-clinically, we believe they are applicable to murine models for VWD and Hemophilia as well and could be modified to use higher frequency equipment specifically designed for small animal imaging.

\section{Clinical ARFI-Monitored Hemostatic Challenge}

Using methods very similar to those described in its pre-clinical application, the ARFIMonitored Hemostatic Challenge was applied clinically to assess hemostasis at femoral arteriotomy [39]. Twenty adult patient volunteers undergoing percutaneous diagnostic cardiac catheterization requiring femoral artery access were enrolled from the Cardiac Catheterization Laboratory in UNC Hospitals. The ultra-sound scanner was wheeled to the patient bed-side after completion of the catheterization procedure, and the imaging transducer was centered above the femoral arteriotomy, with the puncture path from the skin surface to the arteriotomy in the imaging field of view. The subjects underwent ARFI monitoring at the femoral arteriotomy site for a $15 \mathrm{~min}$ observation period immediately following sheath removal. Ten patients were treated with standard of care manual compression to the femoral arteriotomy alone, and ten were treated with manual compression augmented by a hemostatic dressing reported to decrease times to hemostasis by approximately $37 \%$ [40-42]. Table 3 shows the ARFI-derived BR, calculated over fiveminute intervals, and ARFI-derived TTH in the two patient populations. In both patient groups, ARFI-derived BR decreased with time following sheath removal. Moreover, bleeding rates in each time interval were lower in the group treated with manual compression augmented by a hemostatic dressing compared to manual compression alone. ARFI-derived times to hemostasis were also shorter in the hemostatic dressing group.

The data of Table 3 show that ARFI Monitored Hemostatic Challenge yields outcomes that are consistent with expectation for the use of a hemostastic dressing to augment manual compression: 1) statistically significantly slower bleeding rates, and 2) statistically 
significantly faster time to hemostasis in patients treated with the hemostastic dressing versus standard of care manual compression alone. These preliminary clinical data in nonanticoagulated patients strongly suggest that ARFI monitoring of femoral arteriotomy site in patients receiving anti-platelet and anticoagulant therapy will be a valid assay for bleeding phenotype in patients with acquired bleeding disorders.

\section{Conclusion}

The primary saline bleeding time and the secondary cuticle bleeding time have historically been used to document hemostatic efficacy in vivo in dogs with VWD and hemophilia. Both tests are difficult to reproduce in a given dog and vary greatly among dogs. Preliminary data supports that the ARFI-Monitored Hemostatic Challenge is a viable imaging method for in vivo detection of rate of bleeding and onset of hemostasis following a standardized hemostatic challenge in these animals as well as in humans undergoing femoral arteriotomy. On-going work aims to establish the reproducibility and clarity of ARFI-Monitored Hemostatic Challenge end points and describe any associated complications.

\section{References}

1. Rosendaal FR, Smit C, Briet E. Hemophilia treatment in historical perspective: a review of medical and social developments. Ann Hematol. 1991; 62(1):5-15. [Epub 1991/02/01]. [PubMed: 1903310]

2. Carcao M, St Louis J, Poon MC, Grunebaum E, Lacroix S, Stain AM, et al. Rituximab for congenital haemophiliacs with inhibitors: a Canadian experience. Haemophilia. 2006; 12(1):7-18. [Epub 2006/01/18]. [PubMed: 16409170]

3. Bidlingmaier C, Bergmann F, Kurnik K. Haemophilia A in two premature infants. Eur J Pediatr. 2005; 164(2):70-2. [Epub 2004/12/08]. [PubMed: 15580358]

4. Oldenburg J, Ananyeva NM, Saenko EL. Molecular basis of haemophilia A. Haemophilia. 2004; 10(Suppl. 4):133-9. [Epub 2004/10/14]. [PubMed: 15479386]

5. Pipe SW, Saint-Remy JM, Walsh CE. New high-technology products for the treatment of haemophilia. Haemophilia. 2004; 10(Suppl. 4):55-63. [Epub 2004/10/14]. [PubMed: 15479373]

6. Quintana-Molina M, Martinez-Bahamonde F, Gonzalez-Garcia E, Romero-Garrido J, VillarCamacho A, Jimenez-Yuste V, et al. Surgery in haemophilic patients with inhibitor: 20 years of experience. Haemophilia. 2004; 10(Suppl. 2):30-40. [Epub 2004/09/24]. [PubMed: 15385044]

7. Tarantino MD, Collins PW, Hay CR, Shapiro AD, Gruppo RA, Berntorp E, et al. Clinical evaluation of an advanced category antihaemophilic factor prepared using a plasma/albumin-free method: pharmacokinetics, efficacy, and safety in previously treated patients with haemophilia A. Haemophilia. 2004; 10(5):428-37. [Epub 2004/09/11]. [PubMed: 15357767]

8. O'Conner, A. Four Drugs Cause Most Hospitalizations in Older Adults. New York Times; Nov 23. 2011

9. Nichols TC, Dillow AM, Franck HW, Merricks EP, Raymer RA, Bellinger DA, et al. Protein replacement therapy and gene transfer in canine models of hemophilia A, hemophilia B, von Willebrand disease, and factor VII deficiency. ILAR J. 2009; 50(2):144-67. [Epub 2009/03/19]. [PubMed: 19293459]

10. Nathwani AC, Tuddenham EG, Rangarajan S, Rosales C, McIntosh J, Linch DC, et al. Adenovirus-associated virus vector-mediated gene transfer in hemophilia B. N Engl J Med. 2011; 365(25):2357-65. [Epub 2011/12/14]. [PubMed: 22149959]

11. Ponder KP. Merry christmas for patients with hemophilia B. N Engl J Med. 2011; 365(25):2424-5. [Epub 2011/12/14]. [PubMed: 22149960]

12. Ingerslev J, Vanek T, Cullic S. Use of recombinant factor VIIa for emergency reversal of anticoagulation. J Postgrad Med. 2007; 53(1):17-22. [PubMed: 17244965]

13. Levi M. Emergency reversal of antithrombotic treatment. Intern Emerg Med. 2009; 4(2):137-45. [PubMed: 19002653] 
14. Birchall J, Stanworth SJ, Duffy MR, Doree CJ, Hyde C. Evidence for the use of recombinant factor VIIa in the prevention and treatment of bleeding in patients without hemophilia. Transfus Med Rev. 2008; 22(3):177-87. [PubMed: 18572094]

15. Rosovsky RP, Crowther MA. What is the evidence for the off-label use of recombinant factor VIIa (rFVIIa) in the acute reversal of warfarin? ASH evidence-based review. Hematology Am Soc Hematol Educ Program. 2008; 2008:36-8. [Epub 2008/12/17].

16. Langdell RD, Wagner RH, Brinkhous KM. Effect of antihemophilic factor on one-stage clotting tests; a presumptive test for hemophilia and a simple one-stage antihemophilic factor assy procedure. J Lab Clin Med. 1953; 41(4):637-47. [Epub 1953/04/01]. [PubMed: 13045017]

17. Lee RI, White PD. A clinical study of the coagulation time of blood. Am J Med Sci. 1913; 145:495-503.

18. Nichols TC, Franck HWG, Franck C, Raymer RA, Merricks EP. Sensitivity of Whole Blood Clotting Time and Activated Partial Thromboplastin Time for Canine Factor IX. J Thromb Haemost accepted for publication.

19. Giles AR, Tinlin S, Greenwood R. A canine model of hemophilic (factor VIII:C deficiency) bleeding. Blood. 1982; 60(3):727-30. [Epub 1982/09/01]. [PubMed: 6809076]

20. Giles AR, Tinlin S, Hoogendoorn H, Fournel MA, Ng P, Pancham N. In vivo characterization of recombinant factor VIII in a canine model of hemophilia A (factor VIII deficiency). Blood. 1988; 72(1):335-9. [Epub 1988/07/01]. [PubMed: 3134068]

21. Kingdon HS, Hassell TM. Hemophilic dog model for evaluating therapeutic effectiveness of plasma protein fractions. Blood. 1981; 58(5):868-72. [Epub 1981/11/01]. [PubMed: 6794675]

22. Brinkhous K, Sandberg H, Widlund L, Read M, Nichols T, Sigman J, et al. Preclinical pharmacology of albumin-free B-domain deleted recombinant factor VIII. Semin Thromb Hemost. 2002; 28(3):269-72. [Epub 2002/07/05]. [PubMed: 12098087]

23. Brinkhous KM, Sandberg H, Garris JB, Mattsson C, Palm M, Griggs T, et al. Purified human factor VIII procoagulant protein: comparative hemostatic response after infusions into hemophilic and von Willebrand disease dogs. Proc Natl Acad Sci U S A. 1985; 82(24):8752-6. [Epub 1985/12/01]. [PubMed: 3936044]

24. Brinkhous KM, Sigman JL, Read MS, Stewart PF, McCarthy KP, Timony GA, et al. Recombinant human factor IX: replacement therapy, prophylaxis, and pharmacokinetics in canine hemophilia B. Blood. 1996; 88(7):2603-10. [Epub 1996/10/01]. [PubMed: 8839853]

25. Rodgers RP, Levin J. A critical reappraisal of the bleeding time. Semin Thromb Hemost. 1990; 16(1):1-20. [Epub 1990/01/01]. [PubMed: 2406907]

26. Jergens AE, Turrentine MA, Kraus KH, Johnson GS. Buccal mucosa bleeding times of healthy dogs and of dogs in various pathologic states, including thrombocytopenia, uremia, and von Willebrand's disease. Am J Vet Res. 1987; 48(9):1337-42. [Epub 1987/09/01]. [PubMed: 3499100]

27. Mertz ET. The anomaly of a normal Duke's and very prolonged saline bleeding time in swine suffering from an inherited bleeding disease. Am J Physiol. 1942; 136:360-2.

28. Nichols TC, Bellinger DA, Merricks EP, Raymer RA, Kloos M, DeFriess N, et al. Porcine and Canine von Willebrand Factor and von Willebrand Disease: Hemostasis, Thrombosis, and Atherosclerosis Studies. Thrombosis. 2010; 2010:11. doi: 10.1155/2010/461238 [Article ID 461238].

29. Brinkhous KM, Hedner U, Garris JB, Diness V, Read MS. Effect of recombinant factor VIIa on the hemostatic defect in dogs with hemophilia A, hemophilia B, and von Willebrand disease. Proc Natl Acad Sci U S A. 1989; 86(4):1382-6. [Epub 1989/02/01]. [PubMed: 2784006]

30. Brinkhous KM, Sandberg H, Garris JB, Mattsson C, Palm M, Griggs TR, et al. Purified human factor VIII procoagulant protein: Comparative hemostatic response after infusions into hemophilic and von Willebrand disease dogs. Proc Natl Acad Sci U S A. 1985; 82:8752-6. [PubMed: 3936044]

31. Brinkhous KM, Sigman JL, Read MS, Stewart PF, McCarthy KP, Timony GA, et al. Recombinant human factor IX: Replacement therapy, prophylaxis, pharmacokinetics, and immunogenicity in canine hemophilia B. Blood. 1996; 88:2603-10. [PubMed: 8839853] 
32. Brinkhous KM, Sandberg H, Widlund L, Read MS, Nichols TC, Sigman J, et al. Preclinical pharmacology of albumin-free B-domain deleted recombinant factor VIII. Semin Thromb Hemost. 2002; 28(3):269-72. [PubMed: 12098087]

33. Brassard JA, Meyers KM. Evaluation of the buccal bleeding time and platelet glass bead retention as assays of hemostasis in the dog: The effects of acetylsalicylic acid, warfarin and von Willebrand factor deficiency. Thromb Haemost. 1991; 65(2):191-5. [PubMed: 2053106]

34. Nichols TC, Bellinger DA, Davis KE, Koch GG, Reddick RL, Read MS, et al. Porcine von Willebrand disease and atherosclerosis. Influence of polymorphism in apolipoprotein B100 genotype. Am J Pathol. 1992; 140(2):403-15. Epub 1992/02/01. [PubMed: 1739133]

35. Nightingale K, Soo MS, Nightingale R, Trahey G. Acoustic radiation force impulse imaging: in vivo demonstration of clinical feasibility. Ultrasound Med Biol. 2002; 28(2):227-35. [Epub 2002/04/09]. [PubMed: 11937286]

36. Scola MR, Nichols TC, Zhu H, Caughey MC, Merricks EP, Raymer RA, et al. ARFI ultrasound monitoring of hemorrhage and hemostasis in vivo in canine von Willeb-rand disease and hemophilia. Ultrasound Med Biol. 2011; 37(12):2126-32. [Epub 2011/10/29]. [PubMed: 22033127]

37. Margaritis P, Roy E, Aljamali MN, Downey HD, Giger U, Zhou S, et al. Successful treatment of canine hemophilia by continuous expression of canine FVIIa. Blood. 2009; 113(16):3682-9. [Epub 2008/12/26]. [PubMed: 19109232]

38. Nichols TC, Bellinger DA, Reddick RL, Smith SV, Koch GG, Davis K, et al. The roles of von Willebrand factor and factor VIII in arterial thrombosis: studies in canine von Willebrand disease and hemophilia A. Blood. 1993; 81(10):2644-51. [Epub 1993/05/15]. [PubMed: 8490173]

39. Behler RH, Scola MR, Nichols TC, Caughey MC, Fisher MW, Zhu H, et al. ARFI ultra-sound for in vivo hemostasis assessment postcardiac catheterization, part II: pilot clinical results. Ultrason Imaging. 2009; 31(3):159-71. [Epub 2009/09/24]. [PubMed: 19771959]

40. Palmer BL, Gantt DS, Lawrence ME, Rajab MH, Dehmer GJ. Effectiveness and safety of manual hemostasis facilitated by the SyvekPatch with one hour of bedrest after coronary angiography using six-French catheters. Am J Cardiol. 2004; 93(1):96-7. [PubMed: 14697477]

41. Nader R, Garcia J, Drushal K, Pesek T. Clinical evaluation of SyvekPatch in patients undergoing interventional, EPS and diagnostic cardiac catheterization procedures. J Invasive Cardiol. 2002; 14:305-7. [PubMed: 12042620]

42. Najjar SF, Healey NA, Healey CM, McGarry T, Khan B, Thatte HS, et al. Evaluation of poly-Nacetyl glucosamine as a hemostatic agent in patients undergoing cardiac catheterization: a doubleblind, randomized study. J Trauma. 2004; 57(1 Suppl):S38-41. [PubMed: 15280750] 


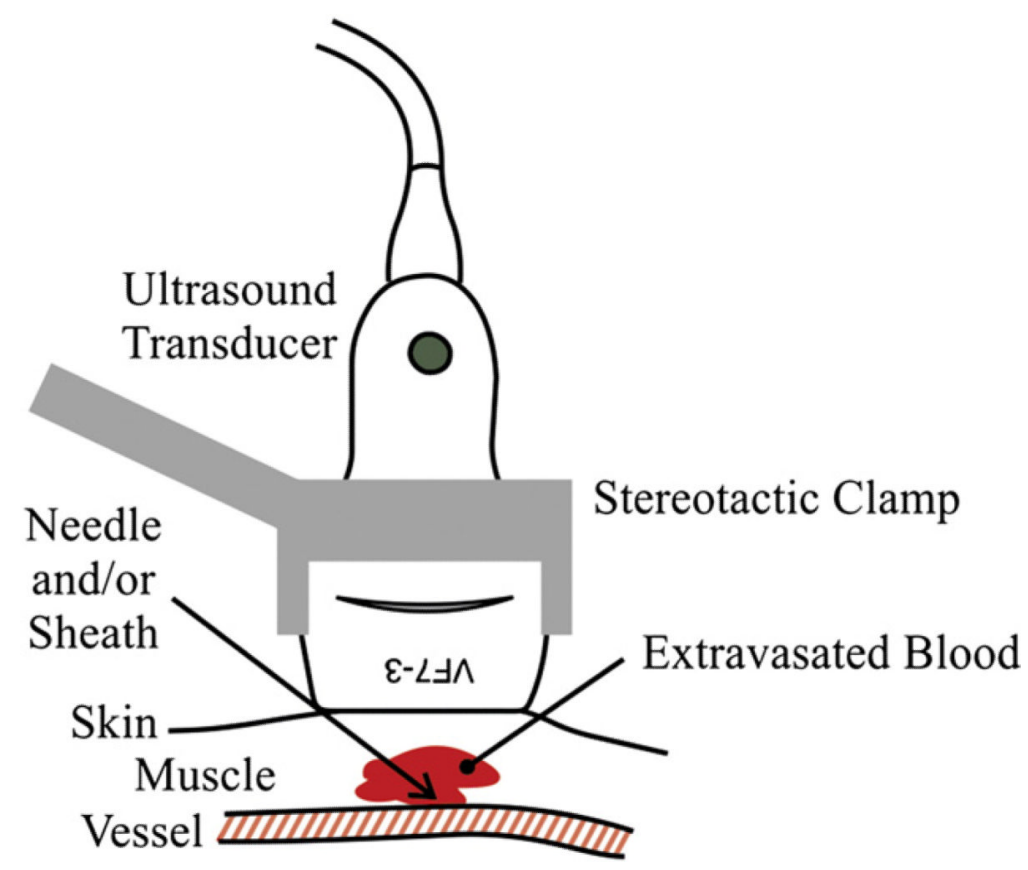

Fig. 1.

The ultrasound transducer, held in place with a stereotactic clamp, is positioned above a peripheral vessel. 


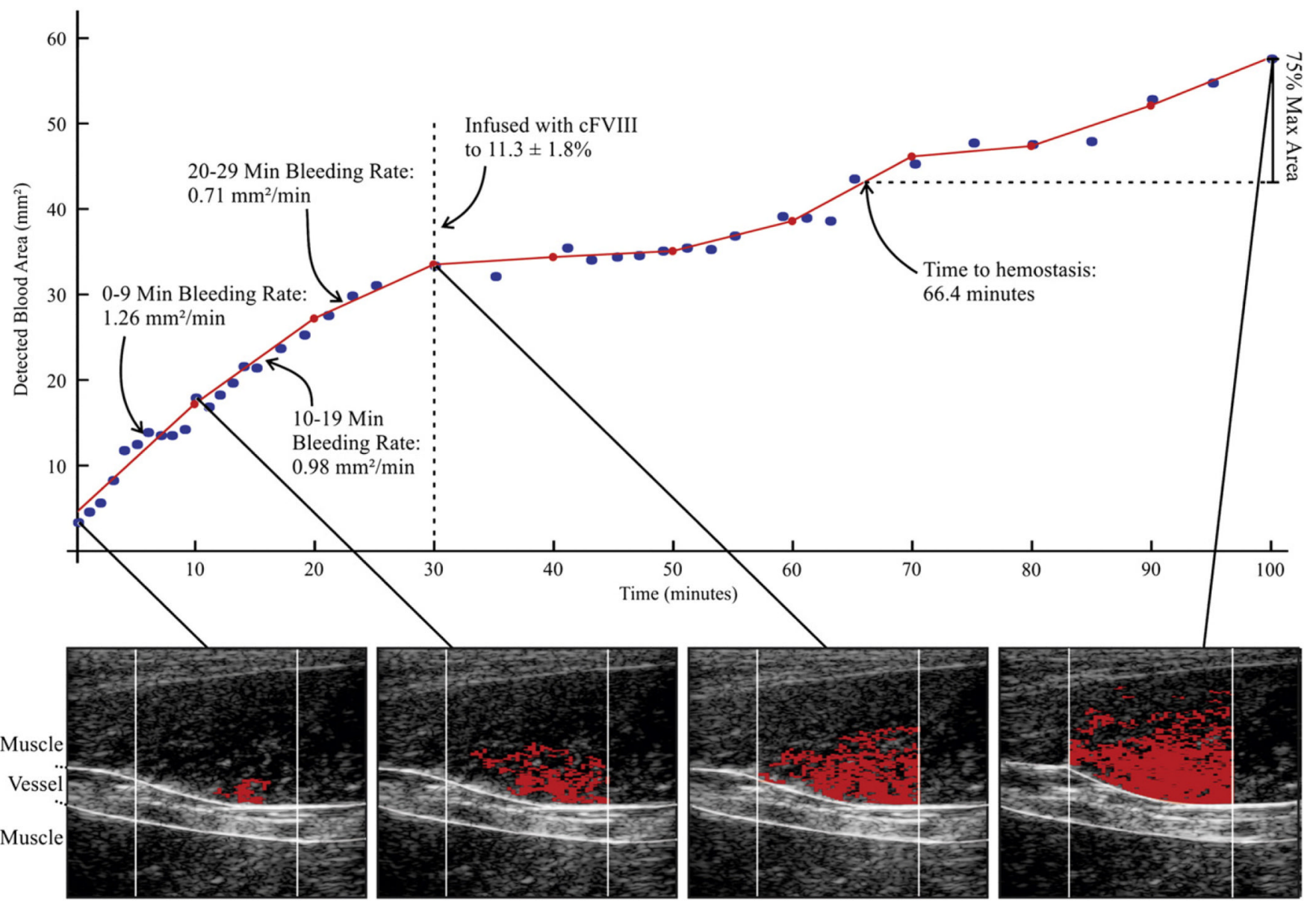

Fig. 2.

Methods of Bleeding Rate and Time to Hemostasis Estimation using ARFI-Monitored Hemostatic Challenge - (bottom row) In serially acquired ARFI frames in a naïve hemophilia A dog, hemorrhage pixels (red) are identified [36] and associated cross-sectional area (CSA) calculated. (top graph) CSA is plotted versus time of image acquisition (blue dots). The CSA plot is subdivided into (in this example, $10 \mathrm{~min}$ ) intervals, and a line is fit using a linear least-squares regression approximation (red lines). The slopes of the lines are recorded as the bleeding rate $[\mathrm{mm} 2 / \mathrm{min}]$. Time to hemostasis onset is estimated as time to $75 \%$ of the maximum observed hemorrhage CSA. FVIII was given at $30 \mathrm{~min}$ to $11.3 \pm 1.8 \%$ of normal. 


\section{Table 1}

Lack of Reproducibility of the Primary Saline Bleeding Time ( $\left.1^{\circ} \mathrm{SBT}\right)$ in VWD dogs (a) and of the Secondary Cuticle Bleeding Times $\left(2^{\circ} \mathrm{CBT}\right)$ in Hemophilia A Dogs (b).

\begin{tabular}{lcccc}
\hline & >15 min on 1st try & Reproducible & Excessive Bleeding & Unclear endpoints \\
\hline a. VWD dogs $(\mathrm{n}=11)$ & $7 / 11$ & $4 / 7$ & $4 / 11$ & $7 / 11$ \\
b. Hemophilia A $(\mathrm{n}=20)$ & $8 / 20$ & $4 / 8$ & $4 / 20$ & $8 / 20$ \\
\hline
\end{tabular}

* bleeding slowed to almost zero then suddenly increased during observation period. 
Table 2

ARFI-Monitored Hemostatic Challenge Bleeding Rates \& Times to Hemostasis by Dog Phenotype.

\begin{tabular}{lccc}
\hline Hemostasis Phenotype Dog Genotype & n & Mean Bleeding Rate $\left(\mathbf{m m}^{2} / \mathbf{m i n}\right)$ & Mean Time to Hemostasis (min) \\
\hline Normal Hemostasis & 6 & $0.62 \pm 0.33^{\#}$ & $17.52 \pm 6.05^{\S}$ \\
$\quad$ 1. Normal & & & \\
Abnormal Secondary Hemostasis & 8 & $0.86 \pm 0.34^{\#}$ & $>30^{\S}$ \\
$\quad$ 2. Naïve Hemophilia A & 8 & $0.61 \pm 0.30^{\#}$ & $28.40 \pm 12.39^{\S}$ \\
3. Hemophilia A+FVIII to $11.3 \pm 1.8 \%$ of normal & $3^{\dagger}$ & $0.62 \pm 0.29^{\#}$ & $26.09 \pm 5.58^{\S}$ \\
4. Hemophilia A expressing FVIIa at $1.3-2 \mu \mathrm{g} / \mathrm{ml}[37]$ & & & $23.98 \pm 9.90^{\S}$ \\
Abnormal Primary Hemostasis & 6 & $1.72 \pm 0.43^{\#}$ & $14.64 \pm 9.32^{\S}$ \\
5. Naïve VWD & 4 & $0.47 \pm 0.35^{\#}$ & \\
6. VWD+VWF to $7.1 \pm 1.7 \%$ of normal VWF:Ag & &
\end{tabular}

* ARFI Time to Hemostasis is defined as that required to bleed $75 \%$ of the total detected area of hemorrhage.

${ }^{\dagger}$ Mean from two separate procedures on each dog. No additional plasma therapy was required in these dogs.

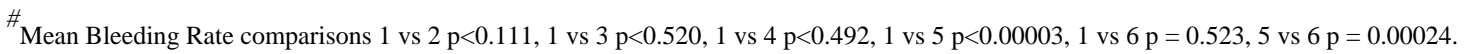

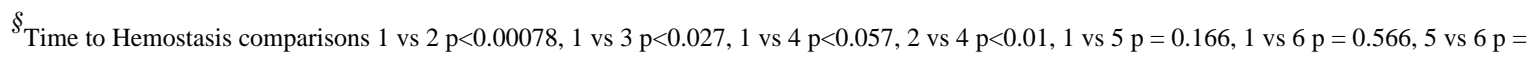
0.137 . 


\section{Table 3}

Clinical Bleeding Rates \& Time to Hemostasis from ARFI-Monitored Hemostatic Challenge by Treatment Type.

\begin{tabular}{|c|c|c|c|c|c|}
\hline \multirow[t]{2}{*}{ Group } & \multirow[t]{2}{*}{$\mathbf{n}$} & \multicolumn{3}{|c|}{ Bleeding Rate $\left(\mathrm{mm}^{2} / \mathrm{min}\right)$} & \multirow{2}{*}{ Time To Hemostasis (min) (4) } \\
\hline & & 0-4 Min (1) & 5-9 Min (2) & 10-14 Min (3) & \\
\hline Manual compression & 10 & $11.15 \pm 6.26^{*}, \psi$ & $8.15 \pm 5.55^{*}, \psi$ & $4.84 \pm 2.71^{*}, \psi$ & $13.00 \pm 1.56^{\psi}$ \\
\hline Hemostatic dressing & $9^{\dagger}$ & $5.44 \pm 3.64^{\xi, \psi}$ & $1.43 \pm 1.9^{\xi, \psi}$ & $-1.93 \pm 2.36^{\xi, \psi}$ & $9.44 \pm 3.09^{\psi}$ \\
\hline \multicolumn{6}{|c|}{ within manual compression group comparisons: 1 vs 2 p<0.136; 1 vs 3 p<0.006. } \\
\hline \multicolumn{6}{|c|}{$\xi$ within hemostasis dressing group comparisons: 1 vs 2 p<0.006; 1 vs 3 p $<0.00009$} \\
\hline \multicolumn{6}{|c|}{$\psi$ between group comparisons: $1: 1 \mathrm{p}<0.013 ; 2: 2 \mathrm{p}<0.02 ; 3: 3 \mathrm{p}<0.00001 ; 4: 4 \mathrm{p}<0.0046$} \\
\hline
\end{tabular}

